\title{
Erratum to: Observing Interactions between Children and Adolescents and their Parents: The Effects of Anxiety Disorder and age
}

\author{
Polly Waite ${ }^{1}$ - Cathy Creswell ${ }^{1}$
}

Published online: 25 June 2015

(C) Springer Science+Business Media New York 2015

Erratum to: J Abnorm Child Psychol

DOI 10.1007/s10802-015-0005-Z

In the original published version of this article, effect sizes for Cohen's d for t-tests were incorrectly described as values of $0.02,0.05$ and 0.08 representing small, medium and large effect sizes, respectively, whereas they should have been reported as $0.2,0.5$ and 0.8 representing small, medium and large effect sizes. This error has no implications for the reported results as our interpretation of the data and subsequent discussion used the correct effect size values.

The online version of the original article can be found at http://dx.doi.org/ 10.1007/s10802-015-0005-z.

Polly Waite

p.1.waite@reading.ac.uk

School of Psychology and Clinical Language Sciences, University of Reading, Whiteknights, Reading RG6 6AL, UK 